Original Article

\title{
Real-World Efficacy Data and Predictive Clinical Parameters for Treatment Outcomes in Advanced Esophageal Squamous Cell Carcinoma Treated with Immune Checkpoint Inhibitors
}

\author{
Jwa Hoon Kim ${ }^{1,2}$, Bokyung Ahn' ${ }^{3}$, Seung-Mo Hong ${ }^{3}$, Hwoon-Yong Jung', Do Hoon Kim ${ }^{4}$, Kee Don Choi ${ }^{4}$, Ji Yong Ahn' ${ }^{4}$, Jeong Hoon Lee \\ Hee Kyoung Na ${ }^{4}$, Jong Hoon Kim ${ }^{5}$, Yong-Hee Kim ${ }^{6}$, Hyeong Ryul Kim${ }^{6}$, Hyun Joo Lee ${ }^{7}$, Sung-Bae Kim', Sook Ryun Park ${ }^{1}$ \\ ${ }^{1}$ Department of Oncology, Asan Medical Center, University of Ulsan College of Medicine, Seoul, ${ }^{2}$ Division of Oncology, Department of Internal \\ Medicine, Korea University Anam Hospital, Korea University College of Medicine, Seoul, Departments of ${ }^{3}$ Pathology, ${ }^{4}$ Gastroenterology, ${ }^{5}$ Radiation \\ Oncology, ${ }^{6}$ Thoracic and Cardiovascular Surgery, and ${ }^{7}$ Radiology, Asan Medical Center, University of Ulsan College of Medicine, Seoul, Korea
}

Purpose This study aimed to evaluate the real-world efficacy of immune checkpoint inhibitors (ICls), and to identify clinicolaboratory factors to predict treatment outcomes in patients with advanced esophageal squamous cell carcinoma (ESCC) receiving ICls.

Materials and Methods Sixty patients with metastatic or unresectable ESCC treated with nivolumab ( $n=48$ ) or pembrolizumab ( $n=12)$ as $\geq$ second-line treatment between 2016 and 2019 at Asan Medical Center were included.

Results The median age of the patients was 68 years (range, 52 to 76 years), and $93.3 \%$ were male. Most patients had metastatic disease $(81.7 \%)$ and had been previously treated with fluoropyrimidines, platinum, and taxane. In 53 patients with measurable disease, the overall response rate and disease control rate were $15.1 \%$ and $35.8 \%$, respectively. With a median follow-up duration of 16.0 months, the median progression-free survival (PFS) and overall survival (OS) were 1.9 months (95\% confidence interval [Cl], 1.54 to 2.19$)$ and 6.4 months $(95 \% \mathrm{Cl}, 4.77$ to 8.11$)$, respectively. After multivariate analysis, recent use of antibiotics, low prognostic nutrition index (<35.93), high Glasgow Prognosis Score $(\geq 1)$ at baseline, and $\geq 1$.4-fold increase in neutrophil-to-lymphocyte ratio after one cycle from baseline were significantly unfavorable factors for both PFS and OS. Younger age ( $<65$ years) was a significant factor for unfavorable PFS and hyponatremia (<135 mmol/L) for unfavorable OS.

Conclusion The use of ICls after the failure of chemotherapy showed comparable efficacy in patients with advanced ESCC in real practice; this may be associated with host immune-nutritional status, which could be predicted by clinical and routine laboratory factors.

Key words Esophageal squamous cell carcinoma, Pembrolizumab, Nivolumab, Survival

\section{Introduction}

Esophageal carcinoma is one of the most common cancers worldwide and its prevalence is highest in East Asia [1]. Esophageal squamous cell carcinoma (ESCC) is the predominant histological subtype in East Asia and accounts for more than $90 \%$ of all esophageal carcinomas in Korea [2,3]. Despite the gradual improvement in survival, the 5 -year relative survival rate remains only $37.4 \%$ according to the nationwide cancer statistics in Korea; this is because most of the patients usually have an advanced stage at the time of diagnosis [2]. For unresectable or metastatic esophageal cancer, palliative chemotherapy is recommended and the most commonly used first-line regimens are based on a combination of fluoropyrimidine and platinum [4], while taxane and irinotecan have also shown antitumor activity as salvage therapies $[5,6]$. Nevertheless, the median survival for patients with unresectable or metastatic esophageal carcinoma remains 8-10 months, and the development of novel therapeutic agents are urgently required.

Following several phase I/II studies showing promising results of immune checkpoint inhibitors (ICIs) against programmed cell death 1 (PD-1), including pembrolizumab and nivolumab [7-9], the phase III KEYNOTE-181 [10] and ATTRACTION-3 [11] trials have demonstrated better efficacy and safety with ICIs in patients with esophageal carcinoma after failure of first-line therapy compared with conventional chemotherapy. In the KEYNOTE-181 trial, two thirds of patients had ESCC subtype, and pembrolizumab significantly improved the overall survival (OS) in patients with programmed cell death ligand 1 (PD-L1) positive tumors (defined as a combined positive score [CPS] $\geq 10$ ) compared with conventional chemotherapy with docetaxel, paclitaxel, or irinotecan. The ATTRACTION-3 trial enrolled

Correspondence: Sook Ryun Park

Department of Oncology, Asan Medical Center, University of Ulsan College of Medicine, 88 Olympic-ro 43-gil, Songpa-gu, Seoul 05505, Korea

Tel: 82-2-3010-3206 Fax: 82-2-3010-6961 E-mail: srpark@amc.seoul.kr

Received November 14, 2020 Accepted June 15, 2021 Published Online June 23, 2021 
only patients with ESCC and nivolumab was demonstrated to significantly improve OS, regardless of PD-L1 status, compared with conventional chemotherapy of docetaxel or paclitaxel. Based on these results, pembrolizumab and nivolumab were approved by the U.S. Food and Drug Administration for $\geq$ second-line treatment in patients with advanced ESCC. Besides survival benefits, ICIs demonstrated better safety than cytotoxic chemotherapy (treatment-related grade $\geq$ 3 adverse events: $18.2 \%$ for pembrolizumab vs. $40.9 \%$ for chemotherapy and $18 \%$ for nivolumab vs. $63 \%$ for chemotherapy); this is particularly important for patients with esophageal cancer seen in routine clinical practice because they are commonly elderly with malnutrition and multiple comorbidities [12].

However, the clinical benefit of ICIs may be limited to only a small portion of patients with advanced esophageal carcinoma, and a subset of patients might experience more rapid tumor progression during the first several months of ICIs compared with chemotherapy as observed in the ATTRACTION-3 study. This suggests the importance of identifying biomarkers to predict which patients could benefit from ICIs. Therefore, this study aimed to evaluate the real-world efficacy of ICIs and to identify clinicolaboratory factors to predict treatment outcomes in patients with advanced ESCC receiving ICIs.

\section{Materials and Methods}

\section{Patients}

Using the electronic medical records database system, a total of 99 patients were identified based on the diagnosis of ESCC and administration history of ICIs between 2015 and 2019 at Asan Medical Center, Seoul, Republic of Korea. Among them, nine patients were given ICIs for other malignancies along with the past history of early ESCC, 10 patients were treated with ICIs as neoadjuvant or adjuvant therapy, and 20 patients were treated with blinded drugs (ICIs vs. placebo) in clinical trials for ESCC. Excluding those patients, 60 patients with metastatic or unresectable ESCC treated with nivolumab $(\mathrm{n}=48)$ or pembrolizumab $(\mathrm{n}=12)$ as $\geq$ second-line therapy between May 2016 to December 2019 were included in this study. Patients received nivolumab $3 \mathrm{mg} / \mathrm{kg}$ intravenously every 2 weeks or pembrolizumab $2 \mathrm{mg} / \mathrm{kg}$ intravenously every 3 weeks until disease progression, unacceptable toxicity, or patients refusal. The tumor response was assessed using computed tomography every 6 to 8 weeks based on Response Evaluation Criteria in Solid Tumors (RECIST) criteria ver. 1.1 [13].

\section{Data collection}

Clinical and laboratory data were collected, and baseline characteristics included the following: patient demographics (age, sex, smoking and alcohol history, and Eastern Cooperative Oncology Group [ECOG] performance status), disease status (initially metastatic, recurrent metastatic, recurrent localized unresectable, or localized unresectable), sites of metastasis (lymph node, liver, lung, bone, peritoneum, and others), the recent use of antibiotics, which was defined as at least one dose of antibiotics within a month before ICI administration, and laboratory findings. Peripheral blood tests were performed at baseline before initiation of ICIs and before each treatment cycle, from which the absolute neutrophil count, absolute lymphocyte count, hemoglobin, platelet, sodium, albumin, lactate dehydrogenase (LDH), and $\mathrm{C}$-reactive protein (CRP) values were collected. The neutrophil-to-lymphocyte ratio (NLR) was calculated by dividing the absolute neutrophil count by the absolute lymphocyte count, and the platelet-to-lymphocyte ratio (PLR) was calculated by dividing the absolute platelet count by the absolute lymphocyte count [14]. The prognostic nutrition index (PNI) was calculated using the following formula: $10 \times$ serum albu$\min (\mathrm{g} / \mathrm{dL})+0.005 \times$ total lymphocyte count $\left(/ \mathrm{mm}^{3}\right)$ [15]. The Glasgow Prognosis Score (GPS) was calculated as follows: patients with increased CRP (> $10 \mathrm{mg} / \mathrm{L}$ ) and hypoalbuminemia $(<3.5 \mathrm{~g} / \mathrm{dL})$ were allocated a score of 2 , patients with only one of these laboratory abnormalities were allocated a score of 1 , and patients with neither were allocated a score of 0 [16].

\section{Available tissue collection}

Available archival tissues from primary or metastatic sites were used to evaluate the PD-L1 status. Immunohistochemistry (IHC) staining was performed on a Dako Autostainer Link 48 system (Agilent Technologies, Santa Clara, CA) using a Dako PD-L1 IHC 22C3 pharmDx kit (Agilent Technologies). The level of PD-L1 protein expression was determined using the TPS or CPS, which was calculated as the number of PD-L1-stained cells (TPS; tumor cells or CPS; tumor cells, lymphocytes, and macrophages) divided by the total number of viable tumor cells and multiplied by 100 . Tumor PD-L1 positivity was defined as TPS $\geq 1 \%$ or CPS $\geq 1$ and also analyzed according to the various cutoff values (1, 5, and 10).

\section{Statistical analysis}

Categorical and quantitative data were compared using the chi-square test or Fisher exact test and Mann-Whitney Utest, respectively. The percentage change in laboratory values from baseline was calculated using the following formula: $100 \times$ ([post-cycle 1 values-baseline value]/baseline value) . 
Table 1. Baseline characteristics

\section{Characteristic}

No. $(\%)(\mathrm{n}=60)$

\begin{tabular}{|c|c|}
\hline Age, median (range, yr) & $68(52-76)$ \\
\hline \multicolumn{2}{|l|}{ Sex } \\
\hline Male & $56(93.3)$ \\
\hline Female & $4(6.7)$ \\
\hline \multicolumn{2}{|l|}{ ECOG PS } \\
\hline $0-1$ & $34(56.7)$ \\
\hline$\geq 2$ & $25(41.7)$ \\
\hline Unknown & $1(1.7)$ \\
\hline \multicolumn{2}{|l|}{ Disease setting } \\
\hline Initially metastatic & $20(33.3)$ \\
\hline Recurrent metastatic & $29(48.3)$ \\
\hline Recurrent localized unresectable & $6(10.0)$ \\
\hline Localized unresectable & $5(8.3)$ \\
\hline \multicolumn{2}{|l|}{ Site of metastasis } \\
\hline Liver & $14(23.3)$ \\
\hline Lung & $36(60.0)$ \\
\hline Peritoneum & $4(6.7)$ \\
\hline Bone & $10(16.7)$ \\
\hline Lymph node in chest & $40(66.7)$ \\
\hline Lymph node in abdomen & $26(43.3)$ \\
\hline Others & $13(21.7)$ \\
\hline Prior surgical resection of primary tumor & $30(50.0)$ \\
\hline Prior concurrent chemoradiotherapy & $37(61.7)$ \\
\hline \multicolumn{2}{|l|}{ Prior palliative chemotherapy } \\
\hline Fluoropyrimidine & $54(90.0)$ \\
\hline Platinum & $57(95.0)$ \\
\hline Taxane & $44(73.3)$ \\
\hline Irinotecan & $24(40.0)$ \\
\hline Others & $3(5.0)$ \\
\hline $\begin{array}{l}\text { Recent use of antibiotics within } \\
\text { the past month }\end{array}$ & $15(25.0)$ \\
\hline \multicolumn{2}{|l|}{ PD-L1 status ${ }^{\text {a) }}$} \\
\hline TPS $<1 \%$ & $17(41.5)$ \\
\hline $\mathrm{TPS} \geq 1 \%$ & $24(58.5)$ \\
\hline $\mathrm{TPS} \geq 5 \%$ & $21(51.2)$ \\
\hline $\mathrm{TPS} \geq 10 \%$ & $11(26.8)$ \\
\hline $\mathrm{CPS}<1$ & $7(17.1)$ \\
\hline $\mathrm{CPS} \geq 1$ & $34(82.9)$ \\
\hline $\mathrm{CPS} \geq 5$ & $29(70.7)$ \\
\hline $\mathrm{CPS} \geq 10$ & $23(56.1)$ \\
\hline \multicolumn{2}{|l|}{ Treatment line } \\
\hline 2nd line & $21(35.0)$ \\
\hline$\geq 3$ rd line & $39(65.0)$ \\
\hline
\end{tabular}

(Continued)

The cutoff values for low vs. high baseline NLR, PLR, or PNI or the percentage changes of NLR and PLR were determined by receiver operating characteristic curves for early disease progression at the first tumor evaluation. Reference
Table 1. Continued

Characteristic

No. $(\%)(n=60)$

Type of immune checkpoint inhibitors

Nivolumab 48 (80.0)

Pembrolizumab

$12(20.0)$

CPS, Combined Positive Score; ECOG PS, Eastern Cooperative Oncology Group performance status; PD-L1, programmed

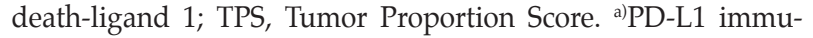
nohistochemistry was stained using a Dako 22C3 pharmDx in 41 available tissues and the patient percentage was calculated among 41 patients.

ranges were used as the cutoff values for other laboratory findings (hemoglobin, sodium, albumin, LDH, and CRP). Progression-free survival (PFS) was calculated from the date of initiation of ICIs to the date of disease progression or death from any cause, whichever occurred first. OS was calculated from the date of initiation of ICIs to the date of death from any cause. Survival was estimated using the Kaplan-Meier method, and the log-rank test was used to compare differences between the curves. Prognostic factors for PFS and OS were analyzed using Cox proportional hazard regression model. A logistic regression model was used to calculate the odds ratios (ORs) of the objective response rate (ORR) (which denoted the proportion of patients with a complete response [CR] or partial response [PR]) and the disease control rate (DCR) (the proportion of patients with a CR, PR, or stable disease [SD]) according to clinical and laboratory factors. Multivariate analysis included factors that were significant $(\mathrm{p}<0.1)$ in the univariate analysis, and the backward elimination model was used in the multivariate analysis. The NLR was used instead of absolute neutrophil or lymphocyte counts, and the GPS and PNI were used instead of CRP and albumin to avoid multicollinearity in multivariate analysis of the Cox proportional hazard regression and logistic regression models. A two-sided p-value $<0.05$ was considered significant, and all statistical analyses were performed using the SPSS ver. 25.0 software package (IBM Corp., Armonk, NY).

\section{Results}

\section{Patient characteristics}

Table 1 summarizes the baseline characteristics of the study patients. Sixty patients received nivolumab $(\mathrm{n}=48,80.0 \%)$ or pembrolizumab $(\mathrm{n}=12,20.0 \%)$ as second-line $(35.0 \%)$ or $\geq$ third-line $(65.0 \%)$ therapy. The median age of the patients was 68 years (range, 52 to 76 years), and $93.3 \%$ of the patients were male. Most patients had metastatic disease $(81.7 \%)$ 
A
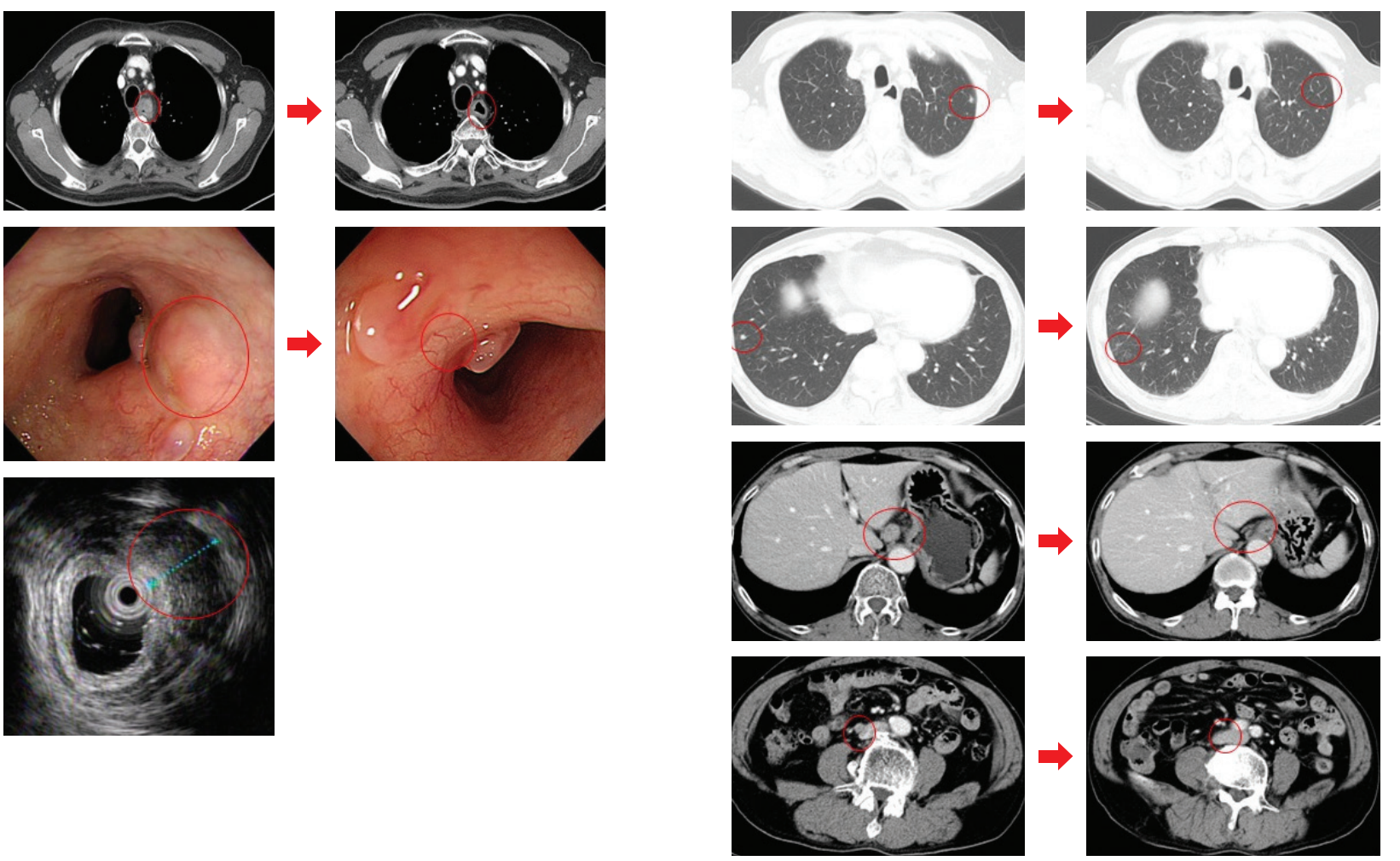

Fig. 1. Representative images of two patients who achieved complete response (CR) with immune check point inhibitors. (A) Esophagogastroduodenoscopy and endoscopic ultrasound images of a 71-year-old man with recurrent localized unresectable esophageal squamous cell carcinoma after chemoradiotherapy who was treated with pembrolizumab. After completion of 2-year pembrolizumab treatment, endoscopically CR with no residual cancer cells on biopsy was confirmed. (B) Computed tomography scans of 61-year-old man with recurrent metastatic esophageal squamous cell carcinoma who was treated with nivolumab. After 6 cycles of nivolumab treatment, metastatic lymph nodes and lung nodules disappeared.

A

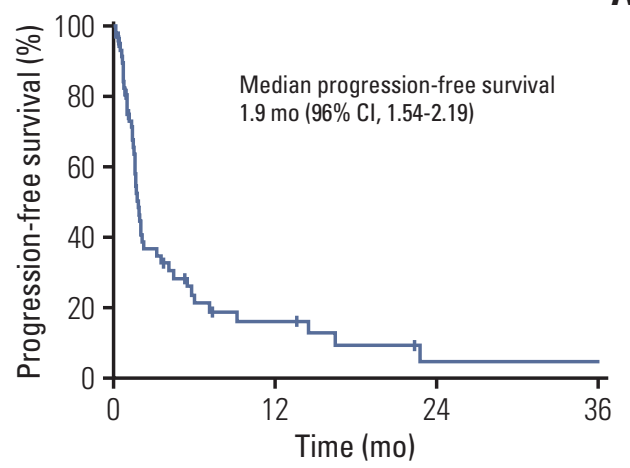

B

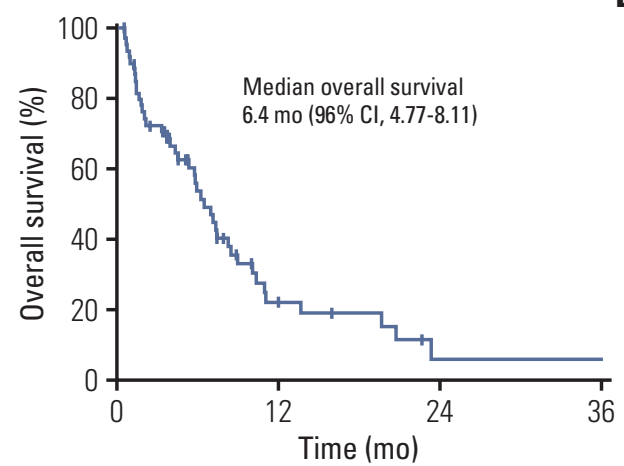

Fig. 2. The Kaplan-Meier curves of progression-free survival (A) and overall survival (B). CI, confidence interval.

and been previously treated with fluoropyrimidines, platinum, and taxane. Fifteen patients $(25.0 \%)$ received at least one dose of antibiotics in the month before ICI administra- tion due to respiratory tract infection $(n=7)$, gastrointestinal and biliary tract infection $(n=6)$, and as a prophylactic $(n=2)$. There were no significant differences in ECOG performance 
Table 2. Univariate and multivariate analysis for progression-free survival

\begin{tabular}{|c|c|c|c|c|c|}
\hline \multirow{2}{*}{ Variable } & \multirow{2}{*}{$\begin{array}{c}\text { Median } \\
\text { values (mo) }\end{array}$} & \multicolumn{2}{|c|}{ Univariate } & \multicolumn{2}{|c|}{ Multivariate $^{a}$} \\
\hline & & HR $(95 \%$ CI $)$ & p-value & HR $(95 \%$ CI) & p-value \\
\hline Age ( $<65$ yr vs. $\geq 65$ yr $)$ & 1.6 vs. 4.1 & $2.24(1.11-4.53)$ & 0.025 & $3.94(1.58-9.85)$ & 0.003 \\
\hline Sex (male vs. female) & 1.8 vs. 1.7 & $1.09(0.39-3.06)$ & 0.869 & - & - \\
\hline Treatment line ( $\geq$ 3rd vs. 2nd) & 1.7 vs. 3.5 & $2.01(1.02-3.98)$ & 0.045 & - & - \\
\hline No. of metastatic organs ( $\geq 2$ vs. 1 ) & 1.6 vs. 3.5 & $1.78(0.95-3.32)$ & 0.072 & - & - \\
\hline ECOG PS ( $\geq 2$ vs. $0-1$ ) & 2.0 vs. 1.7 & $0.87(0.48-1.60)$ & 0.658 & - & - \\
\hline \multicolumn{6}{|l|}{ Site of metastasis } \\
\hline Liver (yes vs. no) & 1.5 vs. 2.0 & $2.86(1.40-5.81)$ & 0.004 & - & - \\
\hline Lung (yes vs. no) & 1.7 vs. 2.0 & $1.11(0.62-2.01)$ & 0.722 & - & - \\
\hline Peritoneum (yes vs. no) & 0.3 vs. 1.9 & $18.34(4.44-75.79)$ & $<0.001$ & - & - \\
\hline Bone (yes vs. no) & 2.0 vs. 1.7 & $0.93(0.41-2.10)$ & 0.865 & - & - \\
\hline Lymph node (yes vs. no) & 1.9 vs. 1.8 & $1.19(0.58-2.48)$ & 0.624 & - & - \\
\hline $\begin{array}{l}\text { Recent use of antibiotics within } \\
\text { the past month (yes vs. no) }\end{array}$ & 0.8 vs. 2.2 & $4.04(1.98-8.24)$ & $<0.001$ & $4.32(1.81-10.32)$ & 0.001 \\
\hline Baseline $\mathrm{Hb}(<12$ g/dL vs. $\geq 12$ g/dL) & 1.7 vs. 6.0 & $2.61(1.08-6.32)$ & 0.033 & - & - \\
\hline $\begin{array}{l}\text { Baseline absolute neutrophil count } \\
(\geq 4,000 \mu / \mathrm{L} \text { vs. }<4,000 \mu / \mathrm{L})\end{array}$ & 0.4 vs. 1.4 & $1.85(1.03-3.35)$ & 0.039 & - & - \\
\hline $\begin{array}{l}\text { Baseline absolute lymphocyte count } \\
(\geq 1,000 \mu / \mathrm{L} \text { vs. }<1,000 \mu / \mathrm{L})\end{array}$ & 1.9 vs. 1.7 & $0.81(0.45-1.46)$ & 0.476 & - & - \\
\hline Baseline NLR ( $\geq 2.71$ vs. $<2.71)$ & 1.6 vs. 4.1 & $2.01(1.08-3.75)$ & 0.028 & - & - \\
\hline Baseline PLR ( $\geq 216.35$ vs. $<216.35$ ) & 1.7 vs. 2.0 & $1.45(0.80-2.64)$ & 0.223 & - & - \\
\hline $\begin{array}{l}\text { Baseline CRP } \\
(>0.6 \mathrm{mg} / \mathrm{dL} \text { vs. } \leq 0.6 \mathrm{mg} / \mathrm{dL})\end{array}$ & 1.6 vs. 4.5 & $2.55(1.23-5.27)$ & 0.011 & - & - \\
\hline Baseline LDH ( $\geq 250$ IU/L vs. $<250$ IU/L) & 1.8 vs. 1.9 & $1.09(0.53-2.23)$ & 0.822 & - & - \\
\hline $\begin{array}{l}\text { Baseline albumin } \\
(<3.5 \mathrm{~g} / \mathrm{dL} \text { vs. } \geq 3.5 \mathrm{~g} / \mathrm{dL})\end{array}$ & 1.7 vs. 2.0 & $1.26(0.68-2.35)$ & 0.462 & - & - \\
\hline $\begin{array}{l}\text { Baseline sodium } \\
(<135 \mathrm{mmol} / \mathrm{L} \mathrm{vs} . \geq 135 \mathrm{mmol} / \mathrm{L})\end{array}$ & 0.8 vs. 2.0 & $5.97(2.53-14.07)$ & $<0.001$ & - & - \\
\hline Baseline GPS (1-2 vs. 0) & 1.6 vs. 4.5 & $2.35(1.19-4.64)$ & 0.014 & $2.43(1.81-10.32)$ & 0.041 \\
\hline Baseline PNI (< 35.93 vs. $\geq 35.93)$ & 1.5 vs. 2.0 & $3.25(1.38-7.67)$ & 0.007 & $4.07(1.29-12.90)$ & 0.017 \\
\hline $\begin{array}{l}\text { 1.4-fold increase of NLR at post-cycle } 1 \\
\text { (yes vs. no) }\end{array}$ & 1.4 vs. 2.1 & $2.23(1.14-4.37)$ & 0.020 & $2.68(1.18-6.09)$ & 0.019 \\
\hline $\begin{array}{l}\text { 1.15-fold increase of PLR at post-cycle } 1 \\
\text { (yes vs. no) }\end{array}$ & 2.2 vs. 1.8 & $1.10(0.60-2.02)$ & 0.754 & - & - \\
\hline Tissue PD-L1 TPS ( $\geq 1 \%$ vs. $<1 \%)^{\text {b) }}$ & 1.6 vs. 1.8 & $1.25(0.62-2.53)$ & 0.535 & - & - \\
\hline Tissue PD-L1 CPS ( $\geq 1$ vs. $<1)^{\text {b) }}$ & 1.7 vs. 1.6 & $0.52(0.22-1.24)$ & 0.137 & - & - \\
\hline
\end{tabular}

CI, confidence interval; CPS, combined positive score; CRP, c-reactive protein; ECOG PS, Eastern Cooperative Oncology Group performance status; GPS, Glasgow Prognostic Score; Hb, hemoglobin; HR, hazard ratio; LDH, lactate dehydrogenase; NLR, neutrophil-lymphocyte ratio; PD-L1, programmed death-ligand 1; PLR, platelet-lymphocyte ratio; PNI, prognostic nutrition index; TPS, tumor proportion score. ${ }^{a}$ Multivariate analysis included significant factors in univariate analysis $(\mathrm{p}<0.1)$, and NLR was used instead of absolute neutrophil

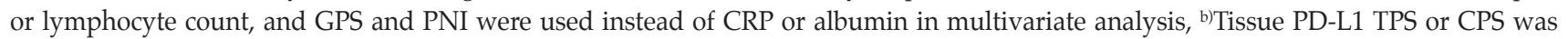
evaluated in 41 available tissues.

status ( $\geq 2)(36.8 \%$ vs. $45.0 \%)$ or hypoalbuminemia (42.1\% vs. $39.0 \%$ ) between patients with and without recent use of antibiotics. Among 60 patients, PD-L1 status was evaluated in 41 patients $(68.3 \%)$ with available tissues receiving nivolumab $(\mathrm{n}=32,53.3 \%)$ or pembrolizumab $(\mathrm{n}=9,15.0 \%)$. The PD-L1 positivity was $58.5 \%$ by TPS $\geq 1 \%$ and $82.9 \%$ by CPS $\geq 1$.

\section{Efficacy outcomes}

S1 Table summarizes the objective tumor response to ICIs. In 53 patients with measurable disease, CR, PR, and SD were achieved in two (3.8\%), six (11.3\%), and 11 (20.8\%) patients, respectively, and 30 patients $(56.6 \%)$ showed progressive disease (PD); the ORR and DCR were $15.1 \%$ (95\% confidence 
Table 3. Univariate and multivariate analysis for overall survival

\begin{tabular}{|c|c|c|c|c|c|}
\hline \multirow{2}{*}{ Variable } & \multirow{2}{*}{$\begin{array}{c}\text { Median } \\
\text { values (mo) }\end{array}$} & \multicolumn{2}{|c|}{ Univariate } & \multicolumn{2}{|c|}{ Multivariate $^{\mathrm{a})}$} \\
\hline & & HR $(95 \% \mathrm{CI})$ & p-value & HR $(95 \% \mathrm{CI})$ & p-value \\
\hline Age (< 65 yr vs. $\geq 65$ yr) & 5.7 vs. 8.4 & $1.77(0.87-3.61)$ & 0.118 & - & - \\
\hline Sex (male vs. female) & 6.2 vs. 10.9 & $4.68(0.64-34.23)$ & 0.129 & - & - \\
\hline Treatment line ( $\geq$ 3rd vs. 2nd) & 5.7 vs. 8.4 & $1.72(0.87-3.41)$ & 0.120 & - & - \\
\hline No. of metastatic organs ( $\geq 2$ vs. 1 ) & 6.4 vs. 10.1 & $2.14(1.10-4.19)$ & 0.022 & - & - \\
\hline ECOG PS ( $\geq 2$ vs. $0-1)$ & 6.9 vs. 5.8 & $1.13(0.60-2.11)$ & 0.704 & - & - \\
\hline \multicolumn{6}{|l|}{ Site of metastasis } \\
\hline Liver (yes vs. no) & 3.7 vs. 7.4 & $2.90(1.41-5.95)$ & 0.004 & - & - \\
\hline Lung (yes vs. no) & 6.9 vs. 6.2 & $1.15(0.62-2.14)$ & 0.661 & - & - \\
\hline Peritoneum (yes vs. no) & 0.7 vs. 7.1 & $20.46(5.26-79.54)$ & $<0.001$ & $4.80(0.97-23.82)$ & 0.055 \\
\hline Bone (yes vs. no) & 3.9 vs. 6.4 & $1.54(0.66-3.57)$ & 0.316 & - & - \\
\hline Lymph node (yes vs. no) & 6.9 vs. 5.7 & $1.34(0.63-2.87)$ & 0.451 & - & - \\
\hline $\begin{array}{l}\text { Recent use of antibiotics within } \\
\text { the past month (yes vs. no) }\end{array}$ & 1.7 vs. 8.2 & $3.88(1.82-8.27)$ & $<0.001$ & $5.14(1.95-13.56)$ & 0.001 \\
\hline Baseline $\mathrm{Hb}(<12 \mathrm{~g} / \mathrm{dL}$ vs. $\geq 12 \mathrm{~g} / \mathrm{dL})$ & 5.8 vs. 10.3 & $3.35(1.28-8.78)$ & 0.014 & - & - \\
\hline $\begin{array}{l}\text { Baseline absolute neutrophil count } \\
(\geq 4,000 \mu / \mathrm{L} \text { vs. }<4,000 \mu / \mathrm{L})\end{array}$ & 3.7 vs. 8.5 & $2.16(1.16-4.00)$ & 0.015 & - & - \\
\hline $\begin{array}{l}\text { Baseline absolute lymphocyte count } \\
(\geq 1,000 \mu / \mathrm{L} \text { vs. }<1,000 \mu / \mathrm{L})\end{array}$ & 7.1 vs. 5.9 & $0.91(0.48-1.73)$ & 0.912 & - & - \\
\hline Baseline NLR ( $\geq 2.71$ vs. $<2.71)$ & 4.5 vs. 10.3 & $2.25(1.17-4.32)$ & 0.015 & - & - \\
\hline Baseline PLR ( $\geq 216.35$ vs. < 216.35) & 4.5 vs. 7.3 & $1.37(0.73-2.56)$ & 0.323 & - & - \\
\hline $\begin{array}{l}\text { Baseline CRP } \\
(>0.6 \mathrm{mg} / \mathrm{dL} \text { vs. } \leq 0.6 \mathrm{mg} / \mathrm{dL})\end{array}$ & 4.3 vs. 8.9 & $3.13(1.45-6.79)$ & 0.004 & - & - \\
\hline Baseline LDH ( $\geq 250$ IU/L vs. $<250$ IU/L) & 6.9 vs. 6.2 & $1.33(0.63-2.79)$ & 0.451 & - & - \\
\hline $\begin{array}{l}\text { Baseline albumin } \\
(<3.5 \mathrm{~g} / \mathrm{dL} \text { vs. } \geq 3.5 \mathrm{~g} / \mathrm{dL})\end{array}$ & 4.5 vs. 8.2 & $1.94(1.04-3.64)$ & 0.038 & - & - \\
\hline $\begin{array}{l}\text { Baseline sodium } \\
\text { (<135 mmol/L vs. } \geq 135 \mathrm{mmol} / \mathrm{L})\end{array}$ & 1.4 vs. 7.4 & $10.25(3.97-26.44)$ & $<0.001$ & $3.27(1.03-10.40)$ & 0.045 \\
\hline Baseline GPS (1-2 vs. 0) & 4.3 vs. 10.1 & $2.93(1.40-6.10)$ & 0.004 & $2.85(1.24-6.56)$ & 0.014 \\
\hline Baseline PNI (< 35.93 vs. $\geq 35.93$ ) & 1.6 vs. 7.1 & $4.61(1.72-12.32)$ & 0.002 & $5.02(1.21-20.76)$ & 0.026 \\
\hline $\begin{array}{l}\text { 1.4-fold increase of NLR at post-cycle } 1 \\
\text { (yes vs. no) }\end{array}$ & 1.8 vs. 8.9 & $3.97(1.96-8.02)$ & $<0.001$ & $3.19(1.46-6.97)$ & 0.004 \\
\hline $\begin{array}{l}\text { 1.15-fold increase of PLR at post-cycle } 1 \\
\text { (yes vs. no) }\end{array}$ & 7.3 vs. 5.9 & $1.15(0.60-2.20)$ & 0.686 & - & - \\
\hline Tissue PD-L1 TPS $(\geq 1 \% \text { vs. }<1 \%)^{\mathrm{b})}$ & 5.3 vs. 8.9 & $1.48(0.72-3.06)$ & 0.289 & - & - \\
\hline Tissue PD-L1 CPS ( $\geq 1$ vs. $<1)^{\mathrm{b})}$ & 5.8 vs. 8.9 & $0.89(0.34-2.34)$ & 0.811 & - & - \\
\hline
\end{tabular}

CI, confidence interval; CPS, combined positive score; CRP, C-reactive protein; ECOG PS, Eastern Cooperative Oncology Group performance status; GPS, Glasgow Prognostic Score; Hb, hemoglobin; HR, hazard ratio; LDH, lactate dehydrogenase; NLR, neutrophil-lymphocyte ratio; PD-L1, programmed death-ligand 1; PLR, platelet-lymphocyte ratio; PNI, prognostic nutrition index; TPS, tumor proportion score. ${ }^{a}$ Multivariate analysis included significant factors in univariate analysis $(\mathrm{p}<0.1)$, and NLR was used instead of absolute neutrophil

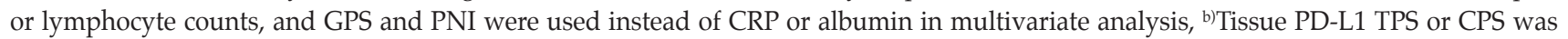
evaluated in 41 available tissues.

interval [CI], 7.6 to 27.3 ) and $35.8 \%$ (95\% CI, 24.3 to 49.3 ). Representative images of two CR responders are shown in Fig. 1. In seven patients with non-measurable disease, four (57.1\%) had non-CR/non-PD and two (28.6\%) had PD; DCR was $57.1 \%$ (95\% CI, 25.0 to 84.3 ). At a median follow-up duration of 16.0 months (95\% CI, 7.4 to 24.5), the median PFS and OS were 1.9 months (95\% CI, 1.54 to 2.19) and 6.4 months (95\% CI, 4.77 to 8.11), respectively (Fig. 2).

There were no significant differences in ORR and DCR between nivolumab and pembrolizumab treatment in 53 patients with measurable disease (ORR, $15.9 \%$ vs. $11.1 \%$ $11.1 \%$; $>0.99$ and DCR, $31.8 \%$ vs. $55.6 \% ; \mathrm{p}=0.282$ ). There were 
A
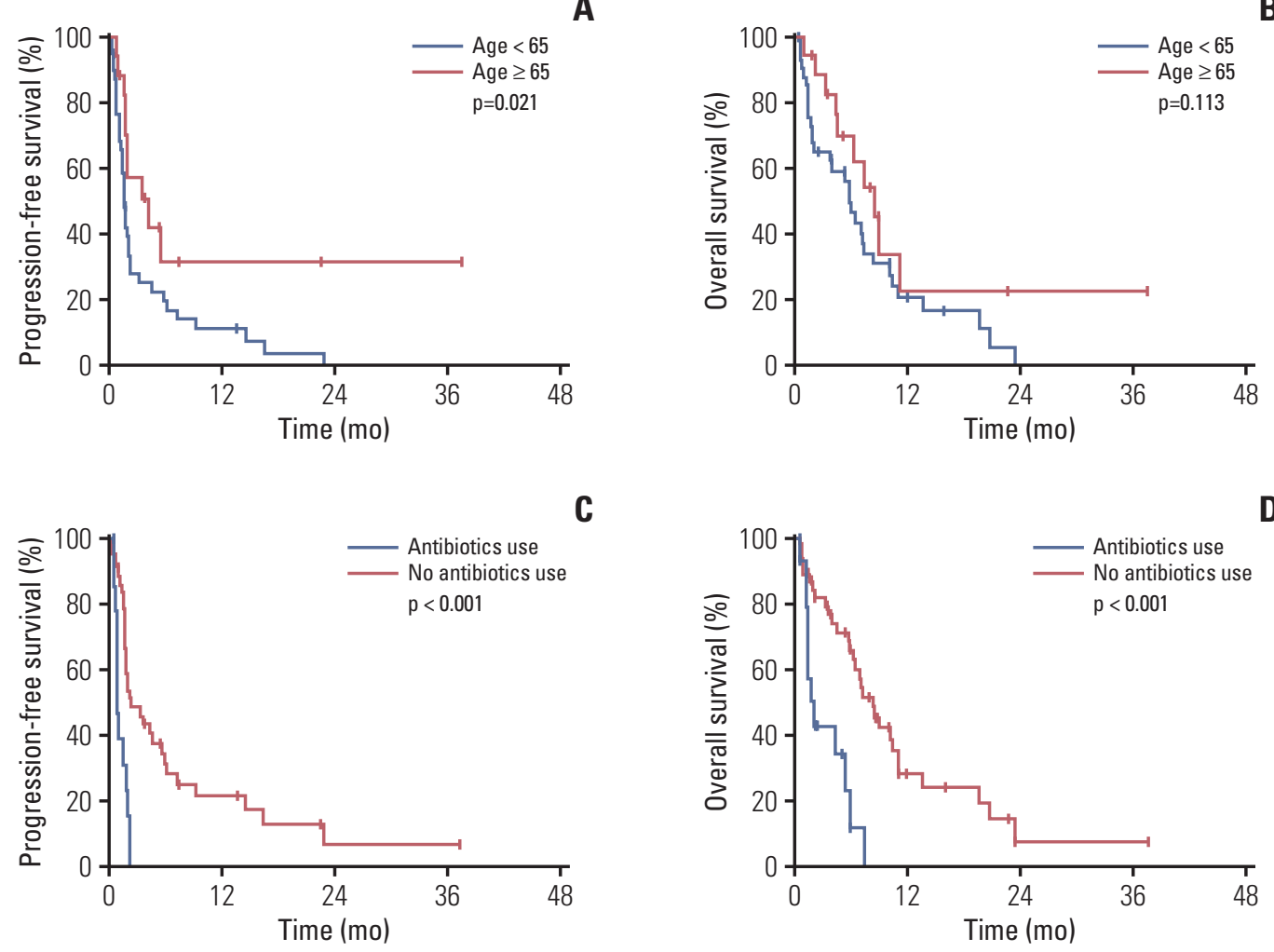

C
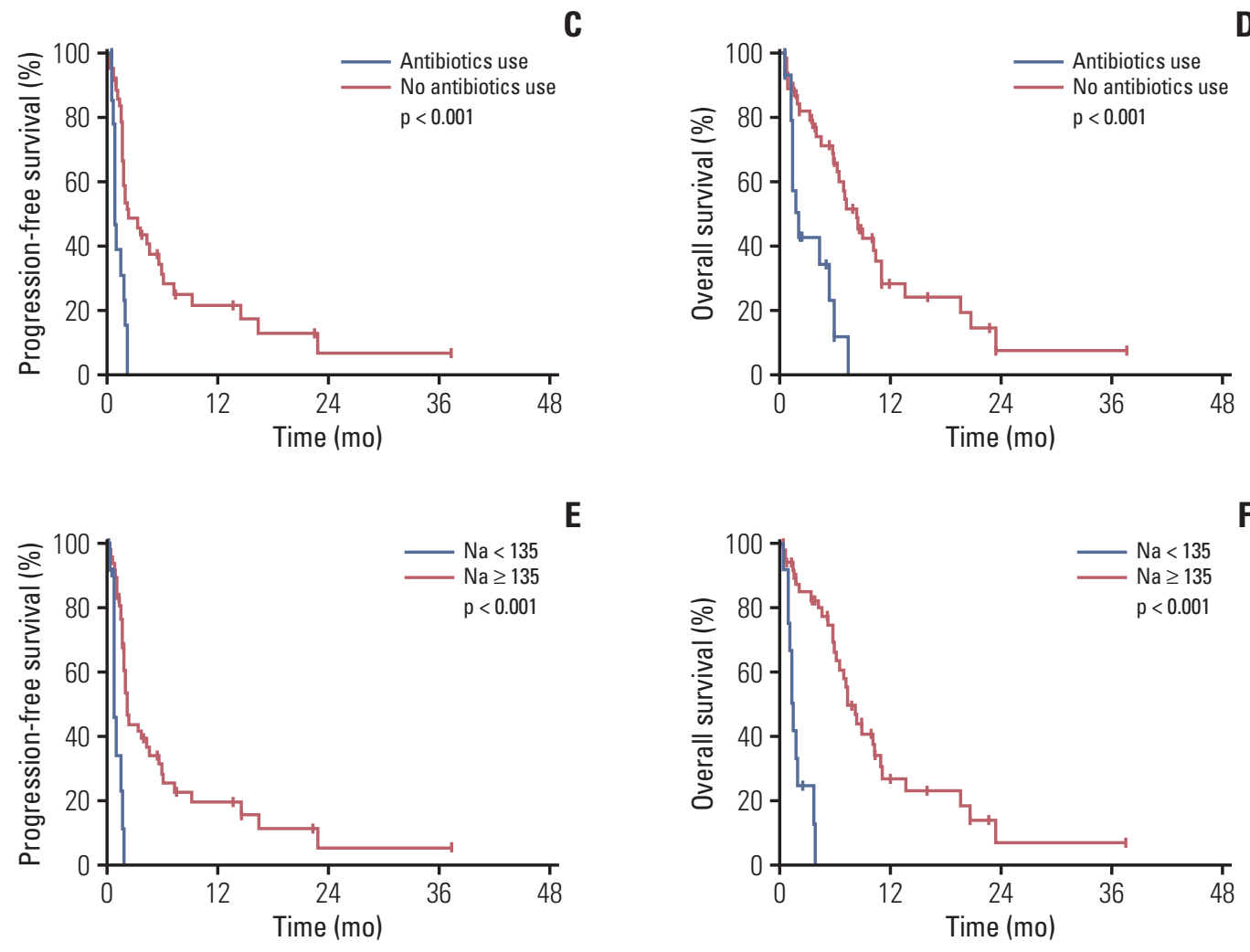

E
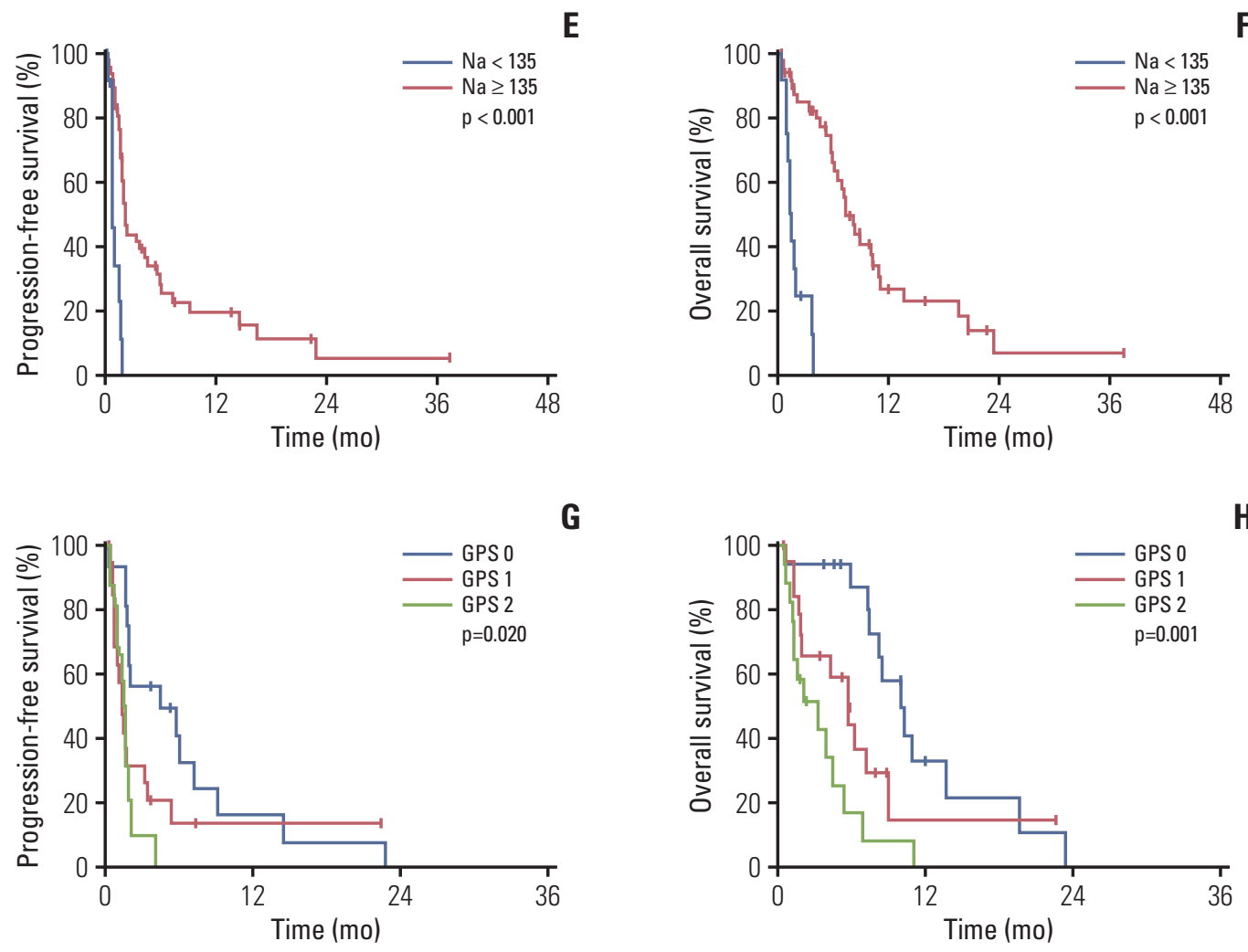

G

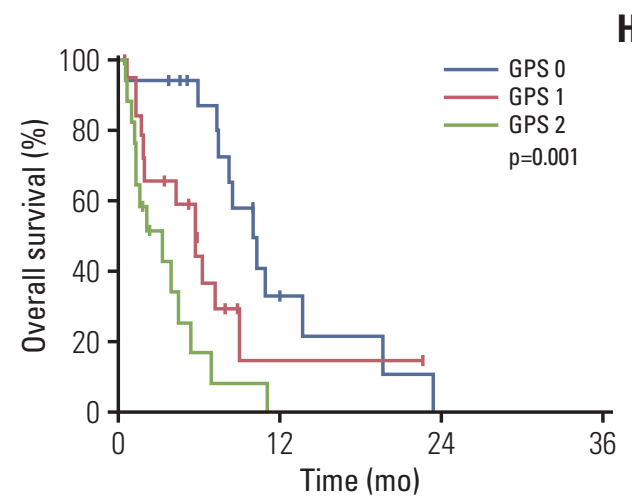

Fig. 3. (A-L) The Kaplan-Meier curves of progression-free survival and overall survival according to significant clinicolaboratory factors. GPS, Glasgow Prognosis Score; PNI, prognostic nutrition index. (Continued to the next page) 

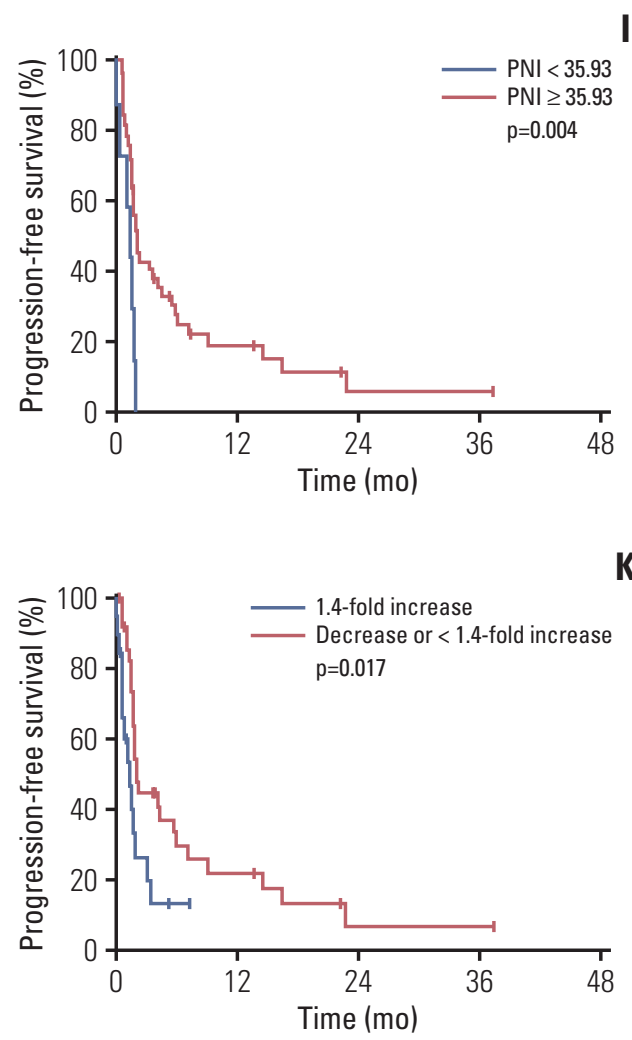

Fig. 3. (Continued from the previous page)

no significant differences in PFS ( $\mathrm{p}=0.138)$ and $\mathrm{OS}(\mathrm{p}=0.633)$ between nivolumab and pembrolizumab treatment.

\section{Analysis of potential clinicolaboratory factors for PFS and OS}

Table 2 summarizes the univariate and multivariate analyses for PFS. After multivariate analyses, younger age $(<65$ years) (hazard ratio [HR], 3.94; 95\% CI, 1.58 to 9.85 ; $\mathrm{p}=0.003$ ), recent use of antibiotics within the past month (HR, 4.32; 95\% CI, 1.81 to 10.32; $\mathrm{p}=0.001)$, baseline GPS $(\geq 1)$ (HR, 2.43; 95\% CI, 1.81 to 10.32; $\mathrm{p}=0.041)$, baseline low PNI $(<35.93)$ (HR, 4.07; 95\% CI, 1.29 to $12.90 ; \mathrm{p}=0.017$ ) and a 1.4 -fold increase in NLR from baseline to post-cycle 1 (HR, 2.68; 95\% CI, 1.18 to $6.09 ; \mathrm{p}=0.019$ ) were significantly associated with poor PFS. Table 3 summarizes univariate and multivariate analyses for OS. After multivariate analyses, recent use of antibiotics in the past month (HR, 5.14; 95\% CI, 1.95 to 13.56; $\mathrm{p}=0.001)$, hyponatremia $(<135 \mathrm{mmol} / \mathrm{L})(\mathrm{HR}, 3.27 ; 95 \% \mathrm{CI}$, 1.03 to 10.40; $p=0.045)$, baseline GPS ( $\geq 1)(\mathrm{HR}, 2.85 ; 95 \% \mathrm{CI}$, 1.24 to $6.56 ; \mathrm{p}=0.014)$, baseline low $\mathrm{PNI}(<35.93)$ (HR, 5.02; 95\% CI, 1.21 to 20.76; $\mathrm{p}=0.026$ ), and a 1.4-fold increase in NLR from baseline to post-cycle 1 (HR, 3.19; 95\% CI, 1.46 to 6.97; $\mathrm{p}=0.004$ ) were significantly associated with poor OS. The Kaplan-Meier curves of PFS and OS according to significant
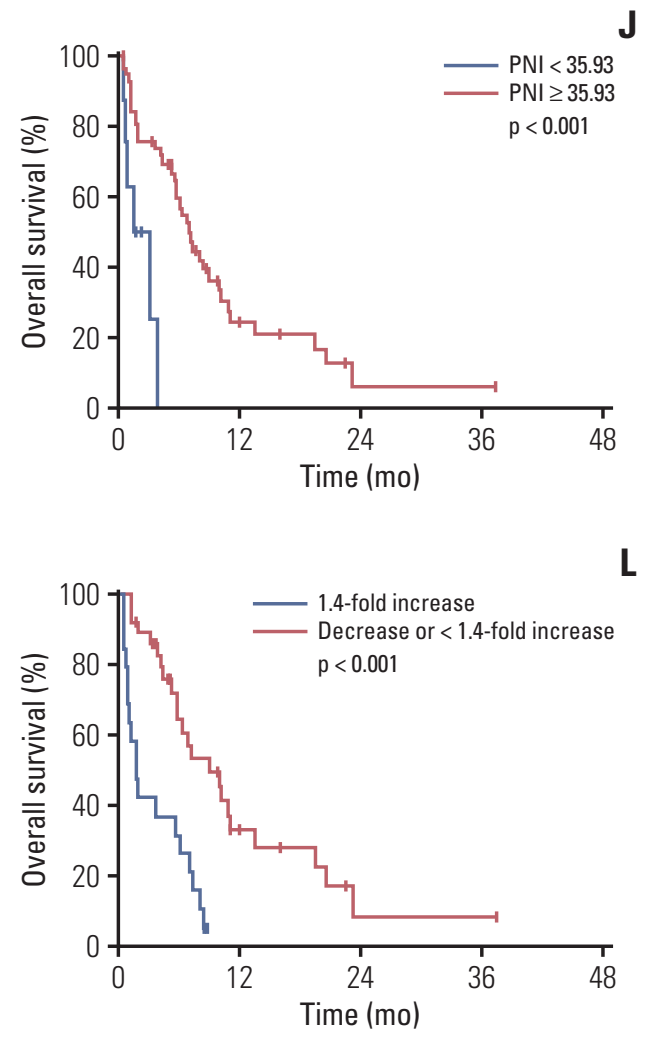

factors in the multivariate analysis are shown in Fig. 2. PFS and OS curves according to clinicolaboratory factors that were significantly associated with PFS or OS only in the univariate analysis are shown in S2 Fig.

\section{Analysis of potential clinicolaboratory factors for objec- tive tumor response}

The univariate and multivariate analyses for ORR according to potential clinicolaboratory factors are summarized in S3 Table. After multivariate logistic regression, baseline GPS $(\geq 1)$ was marginally associated with lower ORR (OR, $0.19 ; \mathrm{p}=0.078)$ and significantly associated with lower DCR $(\mathrm{OR}, 0.20 ; \mathrm{p}=0.027)$. In univariate analysis for ORR and DCR, lower ORR and DCR were associated with the recent use of antibiotics within the past month (ORR, $0 \%$ vs. $19.0 \%$; OR, not calculated and DCR, $15.4 \%$ vs. $50.0 \%$; OR, $0.17 ; \mathrm{p}=0.040$ ), high baseline NLR ( $\geq 2.71$ ) (ORR, 6.1\% vs. 27.3\%; OR, 0.17; $\mathrm{p}=0.046$ and DCR, $30.3 \%$ vs. $59.1 \%$; OR, 0.30; $\mathrm{p}=0.037$ ), increased baseline CRP (>0.6 mg/dL) (ORR, 2.9\% vs. 35.7\%; OR, $0.05 ; p=0.008$ and DCR, $34.3 \%$ vs. $57.1 \%$; OR, $0.39 ; p=$ $0.147)$, and baseline GPS ( $\geq 1)$ (ORR, $6.1 \%$ vs. $25 \%$; OR, 0.19 ; $\mathrm{p}=0.078$ and $\mathrm{DCR}, 30.3 \%$ vs. $62.5 \%$; $\mathrm{OR}, 0.26 ; \mathrm{p}=0.036$ ). 


\section{Analysis of changes in laboratory factors between base- line and post-cycle 1 for PFS and OS}

Among 60 patients, the hemoglobin, albumin, and Na values, as well as the NLR and PLR before the second cycle were available in 55 patients, and the CRP and LDH values before the second cycle were available in 44 patients. Patients with a 1.4-fold increased NLR at post-cycle 1 had significantly worse PFS $(p=0.017)$ and OS $(p<0.001)$ than those with a decreased or $<1.4$-fold NLR at post-cycle 1 (Fig. 3K and L). S4 Fig. shows PFS and OS according to changes in laboratory factors. Patients with hypoalbuminemia at post-cycle 1 had significantly worse PFS $(p=0.038)$ and OS $(p<0.001)$ than those with normal albumin at post-cycle 1. Patients with hyponatremia at baseline or post-cycle 1 had significantly worse PFS $(p<0.001)$ and OS $(p<0.001)$ than those without hyponatremia at both. Patients without increased CRP at both had significantly better PFS $(p=0.023)$ and OS $(p=0.010)$ than other patients. There were no significant differences in PFS and OS according to changes in hemoglobin, LDH, and PLR.

\section{Analysis of tissue PD-L1 status for the efficacy outcomes}

The ORR and DCR were not associated with tissue PDL1 positivity using TPS or CPS (S3 Table). There were also no significant differences in PFS and OS according to PDL1 positivity (S5 Fig.). According to PD-L1 TPS, median PFS and OS were 1.6 months and 5.3 months in PD-L1 TPS $\geq 1 \%$, whereas 1.7 months and 8.9 months in PD-L1 TPS < $1 \%$ ( $\mathrm{p}=0.532$ and $\mathrm{p}=0.285$, respectively). According to PD-L1 CPS, median PFS and OS were 1.7 months and 5.8 months in PD-L1 CPS $\geq 1$, whereas 1.6 months and 8.9 months in PD-L1 CPS $<1$ ( $\mathrm{p}=0.128$ and $\mathrm{p}=0.811$, respectively). Likewise, no significant differences in PFS and OS were observed according to PD-L1 positivity (TPS $\geq 5 \%, 10 \%$ or CPS $\geq 5,10$ ).

\section{Discussion}

Although the treatment options in patients with advanced esophageal carcinoma have expanded through the use of ICIs, the efficacy of ICIs in real practice and the value of biomarkers to identify optimal candidates remains unclear. The current study confirmed the efficacy of ICIs in patients with advanced ESCC after failure of prior chemotherapy in real practice. Moreover, we comprehensively evaluated the prognostic values of potential clinicolaboratory factors for the efficacy of ICIs. Our results showed comparable ORR (15.1\%), median PFS (1.9 months), and median OS (6.4 months) to those of the previous KEYNOTE-181 [10] and ATTRACTION-3 trials [11]. After multivariate analysis, recent use of antibiotics, low PNI $(<35.93)$ and high GPS $(\geq 1)$ at baseline, and $\geq 1.4$-fold increase in NLR from baseline to post-cycle 1 were significantly unfavorable factors for both PFS and OS. Younger age $(<65$ years) was a significant factor for unfavorable PFS and hyponatremia $(<135$ $\mathrm{mmol} / \mathrm{L}$ ) for unfavorable OS.

The KEYNOTE-181 trial [10] compared second-line pembrolizumab with conventional chemotherapy in patients with unresectable or locally advanced esophageal carcinoma (ESCC in two thirds, adenocarcinoma in one third). Among three analysis populations (all patients, patients with ESCC, patients with PD-L1 CPS $\geq 10$ ), only patients with PD-L1 CPS $\geq 10$ showed significant OS benefits with pembrolizumab compared to chemotherapy (HR, 0.69; $\mathrm{p}=0.007)$, whereas patients with ESCC showed a non-significant trend toward better OS with pembrolizumab. Overall PFS was also better with pembrolizumab than chemotherapy ( $\mathrm{HR}, 0.73$; 95\% CI, 0.54 to 0.97$)$. The difference in ORR between patients who received pembrolizumab and those who received chemotherapy was greater in the PD-L1+ population $(21.5 \%$ vs. $6.1 \%, \mathrm{p}<0.001)$ than in the ESCC population $(16.7 \%$ vs. $7.4 \%, \mathrm{p}=0.002)$ or in the total patient population $(13.1 \%$ vs. $6.7 \%, \mathrm{p}=0.004)$. In contrast, in the ATTRACTION-3 trial [11], in which only patients with ESCC were enrolled, secondline nivolumab treatment showed superior OS compared to chemotherapy and survival benefit was not significantly affected by PD-L1 expression status at various cutoffs. Nevertheless, the HR of nivolumab for OS, compared with chemotherapy, was 0.69 (95\% CI, 0.51 to 0.94$)$ in patients with PD-L1 $\geq 1 \%$, whereas 0.84 (95\% CI, 0.62 to 1.14) in patients with PD-L1 < 1\%. Although pembrolizumab and nivolumab demonstrated survival benefits in advanced esophageal carcinoma, not all patients could benefit from them. Rather, a subset of patients experienced more rapid disease progression during an early period of nivolumab treatment compared to those who received chemotherapy; a shorter median PFS with nivolumab than with chemotherapy (1.7 months vs. 3.4 months) in the ATTRACTION-3 trial [11]. Therefore, the identification of biomarkers for the efficacy and / or resistance to ICIs is essential to determine whether an individual patient should be treated with ICIs or chemotherapy as a preferred salvage therapy. Although PD-L1 was suggested as a biomarker for pembrolizumab in the KEYNOTE-181 trial, patients with low or undetectable PD-L1 expression may still gain clinical benefit from pembrolizumab, while a considerable proportion of patients with high PD-L1 expression may not [10]. Furthermore, the PD-L1 positivity was not associated with the efficacy outcomes in our study, even though it was limited by the small sample size. Therefore, the current study focused on clinical and routine laboratory parameters other than PD-L1 to identify predictive biomarkers for ICIs in ESCC. 
Recently, several studies have highlighted the utility of immune prognostic scores to shed light on the importance of routine laboratory parameters because they are easily accessible and sensitive inflammatory- or nutrition-based biomarkers $[17,18]$. Although these biomarkers, including CRP, GPS, PNI, and NLR, have shown prognostic value in various treatment settings for diverse cancers including esophageal cancer [14,18-22], they have gained more attention recently with regard to immune-oncology treatment. Our study demonstrated that baseline high GPS and low PNI, which means high CRP and low lymphocyte counts, respectively, and commonly low albumin levels, could be potential predictors for worse PFS and OS in patients with ESSC treated with PD-1 inhibitors. CRP has been known to be elevated by pro-inflammatory cytokines such as interleukin (IL)-1, IL-8, and IL-6, and has a profound suppressive effect on adaptive immunity by impacting both effector $\mathrm{T}$ cells and antigen presentation. Moreover, CRP is associated with a poor clinical outcome for various cancers treated with ICIs, including melanoma and non-small cell lung cancer (NSCLC) [21]. Hypoalbuminemia has also been associated with the impairment of systemic cell-mediated immune responses, such as macrophage activation and granuloma formation, as well as with poor prognosis in patients with cancer receiving various treatments $[17,18,20]$. Furthermore, a baseline peripheral blood absolute lymphocyte count $\geq 1,000 / \mu$ L has been shown to be significantly associated with favorable PFS (HR, 0.55; $\mathrm{p}=0.04)$ and $\mathrm{OS}(\mathrm{HR}, 0.36 ; \mathrm{p}=0.03)$ in patients with NSCLC treated with nivolumab [23]. Besides each blood-based marker, their combination indexes such as GPS, PNI, and NLR have also shown predictive value for ICIs. In our study, the GPS and PNI had better performance in predicting treatment outcomes than CRP, albumin, and absolute lymphocyte counts. Kurosaki et al. [24] reported that a higher GPS was significantly associated with a shorter PFS (median, 3.0 months vs. 1.6 months vs. 1.4 months for a GPS of 0 vs. 1 vs. 2, respectively; $\mathrm{p}=0.005$ ) and OS (median, 11.0 months vs. 5.1 months vs. 2.9 months for a GPS of 0 vs. 1 vs. 2 , respectively; $p<0.001$ ) in 80 patients with advanced gastric cancer treated with nivolumab. Kurosaki et al. [24] also reported that a high baseline NLR, indicating neutrophilia and lymphopenia, and low PNI were significantly associated with worse PFS and OS in 102 patients with advanced NSCLC treated with anti-PD-1 inhibitors (median PFS, 3.2 months for the high-NLR group vs. 7.3 months for the low-NLR group, $\mathrm{p}=0.009$, and 3.3 months for the low-PNI group vs. 6.3 months for the high-PNI group, $\mathrm{p}=0.007$; median OS, 3.7 months for the high-NLR group vs. 9.8 months for the low-NLR group, $\mathrm{p}=0.002$, and 4.2 months for the low-PNI group vs. 11.5 months for the high-PNI group, $\mathrm{p}<0.001$ ) [25]. Interestingly, in our study, the NLR played a role as a predictor of PFS and OS when considered as a change after one cycle of ICIs, but not the baseline value (which was significant in the univariate analysis for PFS and OS, but not in the multivariate analysis). A 1.4-fold increase in the NLR from baseline was significantly associated with poorer PFS and OS. The consensus defining low- and high-NLR is lacking and the cutoff values of NLR have been variably reported by using median or a receiver operating characteristics curve methods; mainly 2-3 (range, 1.2 to 5.0) in ESCC [14]. However, rather than the cutoff value itself, the findings that high NLR above a certain level and its increase from baseline have been considerably associated with poor prognosis are important. The role of an on-treatment increase in the NLR as a poor prognostic factor for ICIs was also reported in other cancer types, including melanoma, NSCLC, and renal cell carcinoma $[26,27]$. Whereas the baseline NLR might indicate the general immune status of a patient, the change in NLR after ICI therapy might indicate the actual process of ICI-induced immune response. The prediction of treatment response to ICI, especially during the very early period, is of interest given the rapid clinical deterioration seen in a considerable number of patients receiving ICIs. Thus, the change in NLR as early as post-cycle 1 could be a valuable clinical tool to predict a systemic immune response in patients treated with ICI. A recent study also suggested that a systemic immune response in patients responding to ICIs can be observed as soon as 14 days after treatment initiation by ${ }^{18} \mathrm{~F}$-fluorodeoxyglucose positron emission tomography, appearing as increased metabolic activity in the spleen [28].

Of note, the current study validated previous findings which showed the negative impact of antibiotic use on the efficacy of ICIs in patients with various cancers including NSCLC, renal cell carcinoma, and melanoma [29,30]. Recently, the gut microbiota has emerged as a key player in cancer immunotherapy via the modulation of the antitumor immune response. The gut microbiota enhance the function of dendritic cells by increasing tumor antigen presentation, cytokine production, recruitment and activation of interferon- $\gamma$-producing tumor-antigen-specific effector $\mathrm{T}$ cells, and trafficking of $\mathrm{CD}^{+}$memory T-cells from mesenteric and draining lymph nodes to the tumor microenvironment, as well as decreasing regulatory $\mathrm{T}$ cells and myeloid derived suppressor cells $[29,30]$. Given that even short-term antibiotics can shift the gut microbiome to long-term alternative dysbiotic states, and their potential harmful effects on cancer immunotherapy, physicians should be judicious with antibiotics use in patients with cancer who are likely to receive ICIs. Although there is concern that patients who recently received antibiotics would have concomitant medical conditions, there were no significant differences in ECOG perfor- 
mance status $(\geq 2)(36.8 \%$ vs. $45.0 \%)$ and hypoalbuminemia $(42.1 \%$ vs. $39.0 \%)$ between patients who received antibiotics and those who did not.

This study has some limitations. First, this study had a limitation in evaluating predictive values as a single-armed design with small number of patients. Second, being a retrospective study, there were missing laboratory values, inducing a risk of potential selection bias. Third, there may be controversies regarding the cutoff values of NLR, PLR, and PNI. Fourth, the evaluation of PD-L1 status was available in only two-thirds of patients with archival tissues and most of them were obtained at the time of diagnosis, not just before ICI administration as 2nd line or $\geq 3$ rd line, which could limit the predictive role of PD-L1 status. However, this study has investigated impact of all potential clinical and laboratory factors on the efficacy of ICIs in patients with advanced ESCC.

In conclusion, the current study confirmed the efficacy of ICIs in patients with advanced ESCC after the failure of prior standard chemotherapy in real practice. The treatment outcomes of ICIs may depend on the host immune-nutritional status, and could be predicted using clinical and routine laboratory factors at baseline and early after treatment initiation.

\section{Electronic Supplementary Material}

Supplementary materials are available at Cancer Research and Treatment website (https:// www.e-crt.org).

\section{Ethical Statement}

The study protocol was approved by the Institutional Review Board of the AMC (study number: 2019-1639) and has been confirmed for waiver of informed consent. This study was conducted in accordance with the Declaration of Helsinki and Good Clinical Practice.

\section{Author Contributions}

Conceived and designed the analysis: Kim JH, Kim SB, Park SR. Collected the data: Kim JH, Ahn B, Hong SM, Jung HY, Kim DH, Choi KD, Ahn JY, Kim SB, Lee JH, Na HK, Kim JH, Kim YH, Kim HR, Lee HJ, Park SR.

Contributed data or analysis tools: Kim JH, Kim SB, Park SR. Performed the analysis: Kim JH, Kim SB, Park SR.

Wrote the paper: Kim JH, Park SR.

\section{Conflicts of Interest}

Conflict of interest relevant to this article was not reported.

\section{Acknowledgments}

This research was supported by the Bio \& Medical Technology Development Program of the National Research Foundation (NRF) \& funded by the Korean government (MSIT) (No.2019M3E5D4064636) and grants from National Cancer Centre, Korea (NCC1911267).

\section{References}

1. Bray F, Ferlay J, Soerjomataram I, Siegel RL, Torre LA, Jemal A. Global cancer statistics 2018: GLOBOCAN estimates of incidence and mortality worldwide for 36 cancers in 185 countries. CA Cancer J Clin. 2018;68:394-424.

2. Jung KW, Won YJ, Kong HJ, Lee ES. Cancer statistics in Korea: incidence, mortality, survival, and prevalence in 2016. Cancer Res Treat. 2019;51:417-30.

3. Shin A, Won YJ, Jung HK, Kong HJ, Jung KW, Oh CM, et al. Trends in incidence and survival of esophageal cancer in Korea: analysis of the Korea Central Cancer Registry Database. J Gastroenterol Hepatol. 2018;33:1961-8.

4. Al-Batran SE, Hartmann JT, Probst S, Schmalenberg H, Hollerbach S, Hofheinz R, et al. Phase III trial in metastatic gastroesophageal adenocarcinoma with fluorouracil, leucovorin plus either oxaliplatin or cisplatin: a study of the Arbeitsgemeinschaft Internistische Onkologie. J Clin Oncol. 2008;26:1435-42.

5. Ford HE, Marshall A, Bridgewater JA, Janowitz T, Coxon FY, Wadsley J, et al. Docetaxel versus active symptom control for refractory oesophagogastric adenocarcinoma (COUGAR-02): an open-label, phase 3 randomised controlled trial. Lancet
Oncol. 2014;15:78-86.

6. Thuss-Patience PC, Kretzschmar A, Bichev D, Deist T, Hinke A, Breithaupt $K$, et al. Survival advantage for irinotecan versus best supportive care as second-line chemotherapy in gastric cancer: a randomised phase III study of the Arbeitsgemeinschaft Internistische Onkologie (AIO). Eur J Cancer. 2011;47:2306-14.

7. Doi T, Piha-Paul SA, Jalal SI, Saraf S, Lunceford J, Koshiji M, et al. Safety and antitumor activity of the anti-programmed death-1 antibody pembrolizumab in patients with advanced esophageal carcinoma. J Clin Oncol. 2018;36:61-7.

8. Shah MA, Kojima T, Hochhauser D, Enzinger P, Raimbourg J, Hollebecque A, et al. Efficacy and safety of pembrolizumab for heavily pretreated patients with advanced, metastatic adenocarcinoma or squamous cell carcinoma of the esophagus: the phase 2 KEYNOTE-180 study. JAMA Oncol. 2019;5:546-50.

9. Kudo T, Hamamoto Y, Kato K, Ura T, Kojima T, Tsushima T, et al. Nivolumab treatment for oesophageal squamous-cell carcinoma: an open-label, multicentre, phase 2 trial. Lancet 
Oncol. 2017;18:631-9.

10. Kojima T, Muro K, Francois E, Hsu CH, Moriwaki T, Kim $\mathrm{SB}$, et al. Pembrolizumab versus chemotherapy as secondline therapy for advanced esophageal cancer: phase III KEYNOTE-181 study. J Clin Oncol. 2019;37:2(4 Suppl):2.

11. Kato K, Cho BC, Takahashi M, Okada M, Lin CY, Chin K, et al. Nivolumab versus chemotherapy in patients with advanced oesophageal squamous cell carcinoma refractory or intolerant to previous chemotherapy (ATTRACTION-3): a multicentre, randomised, open-label, phase 3 trial. Lancet Oncol. 2019;20:1506-17.

12. Hall PS, Swinson D, Waters JS, Wadsley J, Falk S, Roy R, et al. Optimizing chemotherapy for frail and elderly patients (pts) with advanced gastroesophageal cancer (aGOAC): the GO2 phase III trial. J Clin Oncol. 2019;37(15 Suppl):4006.

13. Eisenhauer EA, Therasse P, Bogaerts J, Schwartz LH, Sargent D, Ford R, et al. New response evaluation criteria in solid tumours: revised RECIST guideline (version 1.1). Eur J Cancer. 2009;45:228-47.

14. Chen MF, Chen PT, Kuan FC, Chen WC. The predictive value of pretreatment neutrophil-to-lymphocyte ratio in esophageal squamous cell carcinoma. Ann Surg Oncol. 2019;26:190-9.

15. Onodera T, Goseki N, Kosaki G. Prognostic nutritional index in gastrointestinal surgery of malnourished cancer patients. Nihon Geka Gakkai Zasshi. 1984;85:1001-5.

16. Wang Y, Li P, Li J, Lai Y, Zhou K, Wang X, et al. The prognostic value of pretreatment Glasgow Prognostic Score in patients with esophageal cancer: a meta-analysis. Cancer Manag Res. 2019;11:8181-90.

17. Feng JF, Wang L, Yang X, Chen S. Gustave Roussy Immune Score (GRIm-Score) is a prognostic marker in patients with resectable esophageal squamous cell carcinoma. J Cancer. 2020;11:1334-40.

18. Mezquita L, Auclin E, Ferrara R, Charrier M, Remon J, Planchard $\mathrm{D}$, et al. Association of the lung immune prognostic index with immune checkpoint inhibitor outcomes in patients with advanced non-small cell lung cancer. JAMA Oncol. 2018;4:351-7.

19. Zingg U, Forberger J, Rajcic B, Langton C, Jamieson GG. Association of $\mathrm{C}$-reactive protein levels and long-term survival after neoadjuvant therapy and esophagectomy for esophageal cancer. J Gastrointest Surg. 2010;14:462-9.

20. Okuno T, Wakabayashi M, Kato K, Shinoda M, Katayama H, Igaki H, et al. Esophageal stenosis and the Glasgow Prognostic Score as independent factors of poor prognosis for patients with locally advanced unresectable esophageal cancer treated with chemoradiotherapy (exploratory analysis of JCOG0303). Int J Clin Oncol. 2017;22:1042-9.

21. Oya Y, Yoshida T, Kuroda H, Mikubo M, Kondo C, Shimi$\mathrm{zu}$ J, et al. Predictive clinical parameters for the response of nivolumab in pretreated advanced non-small-cell lung cancer. Oncotarget. 2017;8:103117-28.

22. Li P, Wang X, Lai Y, Zhou K, Tang Y, Che G. The prognostic value of pre-treatment prognostic nutritional index in esophageal squamous cell carcinoma: a meta-analysis. Medicine (Baltimore). 2019;98:e15280.

23. Tanizaki J, Haratani K, Hayashi H, Chiba Y, Nakamura Y, Yonesaka K, et al. Peripheral blood biomarkers associated with clinical outcome in non-small cell lung cancer patients treated with nivolumab. J Thorac Oncol. 2018;13:97-105.

24. Kurosaki T, Kawakami H, Mitani S, Kawabata R, Takahama T, Nonagase Y, et al. Glasgow Prognostic Score (GPS) and tumor response as biomarkers of nivolumab monotherapy in third- or later-line setting for advanced gastric cancer. In Vivo. 2020;34:1921-9.

25. Peng L, Wang Y, Liu F, Qiu X, Zhang X, Fang C, et al. Peripheral blood markers predictive of outcome and immunerelated adverse events in advanced non-small cell lung cancer treated with PD-1 inhibitors. Cancer Immunol Immunother. 2020;69:1813-22.

26. Moller M, Turzer S, Schutte W, Seliger B, Riemann D. Blood immune cell biomarkers in patient with lung cancer undergoing treatment with checkpoint blockade. J Immunother. 2020;43:57-66.

27. Li M, Spakowicz D, Burkart J, Patel S, Husain M, He K, et al. Change in neutrophil to lymphocyte ratio during immunotherapy treatment is a non-linear predictor of patient outcomes in advanced cancers. J Cancer Res Clin Oncol. 2019;145: 2541-6.

28. Seith F, Forschner A, Weide B, Guckel B, Schwartz M, Schwenck J, et al. Is there a link between very early changes of primary and secondary lymphoid organs in (18)F-FDG-PET/ MRI and treatment response to checkpoint inhibitor therapy? J Immunother Cancer. 2020;8:e000656.

29. Yi M, Yu S, Qin S, Liu Q, Xu H, Zhao W, et al. Gut microbiome modulates efficacy of immune checkpoint inhibitors. J Hematol Oncol. 2018;11:47.

30. Gong J, Chehrazi-Raffle A, Placencio-Hickok V, Guan M, Hendifar A, Salgia R. The gut microbiome and response to immune checkpoint inhibitors: preclinical and clinical strategies. Clin Transl Med. 2019;8:9. 